Physics Vol. 1, No. 2, pp. 127-143, 1964. Physics Publishing Co. Printed in the United States.

\title{
THE MAGNETIC PROPERTIES OF SUPERCONDUCTING ALLOYS. II
}

\author{
KAZUMI MAKI \\ Research Institute for Mathematical Sciences, Kyoto University, Kyoto
}

(Received 22 June 1964)

\begin{abstract}
The lower critical field $\mathrm{H}_{\mathrm{c} 1}$ is derived from the microscopic theory for superconducting alloys. It is shown that Abrikosov's structure in the field just above $\mathrm{H}_{\mathrm{c} 1}$ is described by the use of a parameter of which the temperature dependence is obtained. A brief discussion of the gap in the excitation spectrum and the thermodynamical behavior at lower temperatures is given.

Next the effect of the Pauli paramagnetism on the magnetic properties of superconducting alloys is investigated in detail. It is shown that Abrikosov's structure is still described completely by the use of two parameters $\kappa_{1}{ }^{*}(\mathrm{~T})$ and $\kappa_{2}{ }^{*}(\mathrm{~T})$ in the vicinity of the transition point when the phase transition is of the second order. In superconductors having a large Pauli term $\frac{3 \mu}{\mathrm{e} \tau_{\mathrm{tr}} \mathrm{v}^{2}} \geq 1.475$ where $\mu$ is the Bohr magneton, $\tau_{\mathrm{tr}}$ the transport collision time of an electron and $\mathrm{v}$ the fermi velocity, the order of the transition is found to change from the second to the first as temperature decreases.
\end{abstract}

\section{Introduction}

In the first part [1] of this series (which we refer to as I) we have discussed the magnetic properties of superconducting alloys in the vicinity of the second transition: in the field close to the upper critical field $H_{\mathrm{c} 2}$. It was shown that in this region Abrikosov's structure is completely characterized by two parameters $\kappa_{1}(T)$ and $\kappa_{2}(T)$, both of which coincide with $\kappa$ at the critical temperature $T_{c o}$. Here we first concentrate our attention on the magnetic properties of superconducting alloys near the first transition point, where the penetration of magnetic field into a bulk superconductor begins in the form of quantized flux lines. Since the general discussion is involved, we restrict ourselves to the case $\kappa>1$, where the transition to the mixed state is of the second order. When $\kappa>1$, the flux lines are mutually well separated in fields just above the lower critical field $H_{c 1}$ and we may assume: 1$)$ the local variation of $\Delta$ is so gradual that the expansion in powers of $\frac{\tau_{t r} v^{2}}{\Delta_{\infty}} \nabla^{2}\left(\approx 1 \xi_{0} \nabla^{2}\right)$ is possible and 2) the local variation of $\Delta$ is so small that the varying part can be treated as a small perturbation. Insofar as we are interested in the exterior structure of flux lines, both assumptions are quite reasonable. We will see below that, in the case $\kappa>1$, the energy of a single flux line and the mutual energy between neighbouring lines are almost independent of the core structure of the line. The above assumptions are similar to those made by Tewordt [2] and by Werthamer [3] in the derivation of equations for the ordering parameter $\Delta$ and the current $j$, where $\Delta$ can vary from place to place. In the case of alloys where the electronic mean free path is very short we obtain somewhat different expressions for $\Delta$ and $j$. Using these expressions we discuss Abrikosov's structure [4] in the vicinity of the first transition point as well as the temperature dependence of the lower critical field $H_{c 1}$. It is shown that the structure in this region is described by a parameter $\kappa_{3}(T)$ which reduces to $\kappa$ at the critical temperature. Also the field dependence of the gap in the energy spectrum and the specific heat at lower temperatures are discussed. 
Recently Chandrasekhar [5] and Clogston [6] pointed out that the Pauli paramagnetism might play an important role in the resistive transition of some superconductors, which was revealed subsequently in the experimental study of high field superconductivity carried out by Hake and Berlincourt [7]. Extending the discussion given by Chandrasekhar and by Glogston to finite temperatures, Maki and Tsuneto [8] showed that the order of the phase transition changes from the second to the first as the temperature decreases, if the transition is brought about by the energy difference coming from the Pauli susceptibility. A preliminary discussion on the modification of Abrikosov's structure was also given based on the generalized GinzburgLandau equation, containing the Pauli term.

Here we carry out a rather complete study of the effect of Pauli paramagnetism on the magnetic properties of superconducting alloys. Incorporating the Pauli term in the general Gor'kov equation, we derive a set of equations for the ordering parameter $\Delta$ and the current $j(x)$ on the assumption $\Delta$ is small $\left(\Delta / \pi T_{c o} \ll 1\right)$. Those equations are solved by a similar procedure to that given in $\mathrm{I}$.

We find that the peculiar deviation from Abrikosov's theory noted in I is quite common in superconductors having a large Pauli paramagnetism $\left(\frac{3 \mu}{e \tau_{t r} v^{2}}>1.475\right.$ where $\mu$ is the Bohr magneton, $\tau_{t r}$ the collision time of an electron and $v$ the fermi velocity): in those superconductors the order of the transition changes from the second to the first as temperature decreases and at a critical point $\left(\left(T_{1}, H_{1}\right)\right.$ in the $T-H_{c}$ diagram $)$ the jump of the specific heat at the transition diverges as $\left(T-T_{1}\right)^{-1 / 2}$.

\section{The Equations for $\Delta$ and $i$}

In this section we are concerned with the derivation of the equations for the ordering parameter $\Delta$ and the current $j$, which give the basis of the subsequent discussion. As is shown in I the effect of a magnetic field is introduced into the theory by a simple replacement of $\vec{q}$ by $\vec{q}+2 e \vec{A}$. When the varying part of $\Delta(x)$ is small we obtain the following differential equation [9]

$$
\begin{aligned}
\Delta(x) & =\frac{|q| m p_{0}}{2 \pi^{2}} T \sum_{n} \frac{1}{\sqrt{\omega^{2}+\Delta^{2}}+a} \Delta(x), \\
\omega & =2 \pi T(n+1 / 2)
\end{aligned}
$$

where $a=-\frac{\tau_{t r} v^{2}}{6} \nabla^{2}$ or in the presence of fields $a=-\frac{\tau_{t r} v^{2}}{6}(\nabla-2 i e A)^{2}$ and $A$ is the vector potential. The above equation is rewritten in a much simpler form

$$
\left\{\ln \left(\Delta / \Delta_{\infty}\right)+2 \sum_{n=1}^{\infty}(-1)^{n+1} K_{0}\left(\frac{\Delta n}{T}\right)+2 \pi_{2} T \sum_{n}\left(\frac{1}{\sqrt{\omega^{2}+\Delta^{2}}}-\frac{1}{\sqrt{\omega^{2}+\Delta^{2}}+a}\right)\right\} \Delta(x)=0
$$

or expanding in powers of $a^{*}$

$$
\left\{\ln \left(\Delta / \Delta_{\infty}\right)+2 \sum_{n=1}^{\infty}(-1)^{n+1} K_{0}\left(\frac{\Delta n}{T}\right)+\frac{\pi}{2 \Delta} \tan h\left(\frac{\Delta}{2 T}\right) a\right\} \Delta(x)=0
$$

where $\Delta_{\infty}$ is the gap in the energy spectrum at $T=0^{\circ} \mathrm{K}$ and $H=0$ and $K_{0}(z)$ is the modified Bessel function. The expression for the current has already been found by Abrikosov and Gor'kov [10]

$$
\vec{j}=-\frac{\tau_{t r} e^{2}}{m} N \pi \Delta \tan h\left(\frac{\Delta}{2 T}\right) \vec{A}
$$

where $\Delta$ is the gap at finite temperature [11].

*The coefficient of $a$ is in accordance with Werthamer's (Rev. Mod. Phys. 36, 292 (1964)). 


\section{The Beginning of Field Penetration into a Superconductor}

In a detailed study of the solution of the Ginzburg - Landau equation, Abrikosov [4] has found that the magnetic field begins to penetrate into a superconductor in the form of vortex lines carrying unit flux, in a field smaller than the usual thermodynamical critical field $H_{c}$ when $\kappa$ is sufficiently large. This field is called the lower critical field $H_{c 1}$. We follow a similar procedure to that given by Abrikosov, since his analysis does not depend on the form of the equation for $\Delta$ and seems applicable even when the GinzburgLandau equation no longer holds. As the generalization to the case of finite temperatures is easy, we first consider the case of the absolute zero of temperature for definiteness. In order to determine the lower critical field $H_{c 1}$ we calculate the energy of a flux line. According to Abrikosov we treat the problem in cylindrical coordinates where the axis of symmetry is along the magnetic field. Introducing a new quality $Q$ which is equal to the absolute value of $A_{r}-i \nabla \phi / 2 \mathrm{e}$ and putting $\Delta(x)=\Delta_{\infty} \mathrm{e}^{i \phi} f(r)$ we reduce the equations to one dimensional form

$$
(-\ln f) f^{2}+\frac{\pi \tau_{t r} v^{2}}{12 \Delta_{\infty}}\left(-\frac{1}{r} \frac{d}{d r}\left(r \frac{d f}{d r}\right)+4 e^{2} Q^{2} f\right)=0
$$

and the Maxwell equation to

$$
\frac{d}{d r}\left(\frac{1}{r} \frac{d}{d r}(r Q)\right)=\frac{4 \pi^{2} r_{t r} e^{2}}{m} N \Delta_{\infty} f Q
$$

The magnetic field $H$ is given by

$$
H=-\frac{1}{r} \frac{d}{d r}(r Q)
$$

Considering the fact that $f \simeq 1$ everywhere except in a narrow region around the core of a flux line, we solve Equation (5) in term of the modified Bessel function:

$$
Q=\frac{a}{2 e} K_{1}(a r)
$$

where

$$
a=\left(\frac{4 \pi^{2} e^{2} N}{m} \tau_{t r} \Delta_{\infty}\right)^{1 / 2}
$$

Substituting this in Equation (4) we find

$$
f=1-\frac{\pi}{12} \frac{\tau_{t r} v^{2}}{\Delta_{\infty}}\left(a K_{1}(\alpha r)\right)^{2},
$$

for

$$
r \gg\left(\frac{\pi}{12} \frac{\tau_{t r} v^{2}}{\Delta_{\infty}}\right)^{1 / 2} .
$$

Then the energy of a single flux line is given by

$$
\epsilon=\frac{m p_{0}}{2 \pi^{2}} \Delta_{\infty}^{2}\left(2 \pi \int_{0}^{\infty} \frac{1-f^{2}}{2} r d r\right)
$$




$$
\epsilon=\frac{m p_{0}}{12} \Delta_{\infty} \tau_{t r} v^{2} \ln \left(\frac{1}{\pi \mathrm{e} \tau_{t r} v}\left(\frac{3 m}{\pi N}\right)^{1 / 2}\right),
$$

where we cut off the lower limit of the integration at $r_{0}=\left(\frac{\pi}{12} \frac{\tau_{t r} v^{2}}{\Delta_{\infty}}\right)^{1 / 2}$.

The free energy difference in the presence of the flux lines is

$$
\Delta F=F_{1}-\frac{1}{4 \pi} B H,
$$

where $F_{1}=n \epsilon, H$ is the external field and $n$ the density of flux lines (number of lines in a unit area). $B$ is the magnetic induction and is given by

$$
B=\bar{H}=n \int H d S=\frac{\pi}{\mathrm{e}} n
$$

When $\Delta F$ is negative, the superconductor becomes unstable against the flux invasion and the flux lines begin to penetrate. The lower critical field is determined by

$$
n \epsilon-\frac{1}{4 \pi} B H_{c 1}=0
$$

or

$$
H_{\mathrm{c} 1}=4 \mathrm{e} \epsilon=\frac{m p_{0}}{3} \Delta_{\infty} \tau_{t r} e v^{2} \ln \left(\frac{1}{\pi \mathrm{e} \tau_{t r} \mathrm{e}}\left(\frac{3 m}{\pi N}\right)^{1 / 2}\right) .
$$

This expression is rewritten in a more convenient form by the use of a parameter $\kappa_{3}$ :

$$
\begin{aligned}
& \kappa_{3}(0)=\pi\left(\frac{2}{\zeta(3)}\right)^{1 / 2} \kappa=1.53 \kappa \\
& H_{c 1} / \sqrt{2} H_{c}=\frac{1}{2 \kappa_{3}(0)} \ln \kappa_{3}(0) .
\end{aligned}
$$

A similar expression is found by Abrikosov by using the Ginzburg - Landau theory.

This similarity becomes even more salient when we obtain an equation for the magnetic induction. As is seen from Equation (13) $H_{\mathrm{c} 1}$ is independent of $n$, which holds generally when the phase transition is of the second order. When the external field is larger than $H_{c 1}$ a finite number of flux lines penetrates into the bulk and $n$ is determined from the following consideration. As given by Abrikosov the magnetic field $H$ is determined from

$$
\nabla^{2} H-a^{2} H=-\pi a^{2} / e \sum_{m} \delta\left(\vec{r}-\vec{r}_{m}\right)
$$

where the summation runs over the positions of the flux lines. The solution is found as

$$
H=\frac{a^{2}}{2 \mathrm{e}} \sum_{m} K_{0}\left(a\left|\vec{r}-\vec{r}_{m}\right|\right),
$$

and $F_{1}$ is calculated as 


$$
\begin{aligned}
F_{1} & =\overline{\frac{1}{8 \pi} H\left(H-a^{-2} \nabla^{2} H\right)}, \\
& =\frac{n}{2}\left(\frac{a}{2 e}\right)^{2} \sum_{m} K_{0}\left(a\left|r_{m}\right|\right) .
\end{aligned}
$$

For a triangular lattice we have

$$
F_{1}=\frac{\mathrm{e}}{\pi} B \epsilon+\frac{a^{2}}{8 \pi \mathrm{e}} B \sum_{1^{2}+m^{2}+1 m \geq 1} K_{0}\left(a \sqrt{\frac{2 \pi\left(1^{2}+m^{2}+1 m\right)}{\sqrt{3} e B}}\right) .
$$

and for a square lattice

$$
F_{1}=\frac{e}{\pi} B \epsilon+\frac{a^{2}}{8 \pi e} B \sum_{1^{2}+m^{2} \geq 1} K_{0} a\left(\sqrt{\frac{\pi\left(l^{2}+m^{2}\right)}{e B}}\right) .
$$

Maximizing the free energy difference $F_{1}-\frac{1}{4 \pi} B \cdot H$ we obtain an equation for the magnetic induction

$$
\kappa_{3}(0)\left(H-H_{c 1}\right) / H_{c}=\sum_{1, m}\left[2 \kappa_{0}\left(x_{l m}\right)+x_{l m} \kappa_{1}\left(x_{l m}\right)\right]
$$

where

$$
x_{l m}=a \sqrt{\frac{2 \pi\left(l^{2}+m^{2}+l m\right)}{\sqrt{3} e B}}
$$

for a triangular lattice, and

$$
x_{1 m}=a \sqrt{\frac{\pi\left(1^{2}+m^{2}\right)}{e B}}
$$

for a square lattice.

Thus the structure in the field just above the lower critical field is completely described by Abrikosov's theory for all temperatures if one uses a single appropriate parameter $\kappa_{3}$.

At finite temperatures by using Equation $\left(2^{\prime}\right)$ and the expression for the free energy [7] at finite temperatures

$$
F=-\frac{m p_{0}}{2 \pi^{2}} \int_{0}^{\Delta} \Delta^{2} \frac{d h}{d \Delta} d \Delta
$$

where

$$
h=\ln \left(\Delta / \Delta_{\infty}\right)+2 \sum_{n=1}^{\infty}(-1)^{n+1} K_{0}\left(\frac{\Delta n}{T}\right)
$$

we find 


$$
H_{c 1}(T)=\frac{m p_{0}}{3} e \tau_{t r} v^{2} \Delta \tan h \frac{\Delta}{2 T} \ln \kappa_{3}(T),
$$

and

$$
\kappa_{3}(T)=\kappa_{3}(0) \frac{\left(\frac{1}{2} \int_{0} \Delta^{2} \frac{d h}{d \Delta} d \Delta\right)^{1 / 2}}{\Delta \tan h\left(\frac{\Delta}{2 T}\right)}
$$

We note here Equation (26) is rewritten as

$$
H_{\mathrm{c} 1}(T) / \sqrt{2} H_{\mathrm{c}}(T)=\frac{1}{2 \kappa_{3}(T)} \ln \kappa_{3}(T) .
$$

The magnetic induction is also given by Equation (21), if one replaces $H_{c}$ and $a$ by temperature dependent values respectively where

$$
a(T)=\left(\frac{4 \pi^{2} \mathrm{e}^{2} N}{m} \tau_{t r} \Delta \tan h \frac{\Delta}{2 T}\right)^{1 / 2}
$$

The asymptotic form of $\kappa_{3}(T)$ is given as:

$$
\begin{aligned}
\kappa_{3}(T) & =\kappa_{3}(0)\left(1-\frac{2}{3}\left(\frac{\pi T}{\Delta_{\infty}}\right)^{2}\right)^{1 / 2}, \quad \text { for } T \ll T_{\text {co }} \\
& =\kappa\left\{1-\left(\frac{93}{112 \pi^{2}} \frac{\zeta(5)}{\zeta(3)}-\frac{1}{12}\right)\left(\frac{\Delta}{T}\right)^{2}\right\}, \\
& =\kappa\left(1+0.32\left(1-\frac{T}{T_{c o}}\right)\right), \quad \text { for } T_{c o}-T \ll T_{c o} .
\end{aligned}
$$

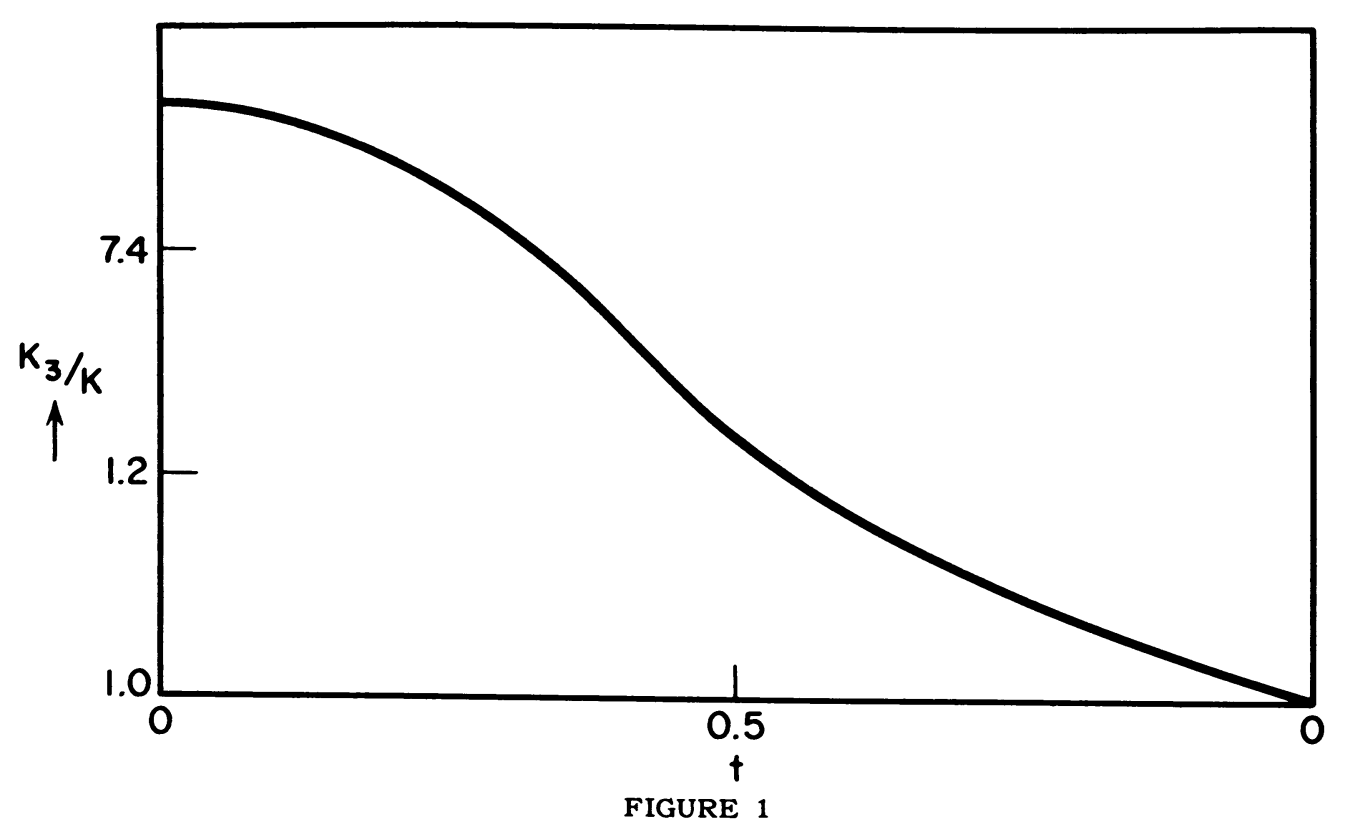

$\kappa_{3} / \kappa$ as a function of the reduced temperature $t=T / T_{c o} \cdot$ 
where $\kappa_{3}(T)$ reduces to $\kappa$ at the critical temperature as it should be. The temperature dependence of $\kappa_{3}(T)$ is numerically calculated and depicted in Fig. 1.

\section{The Gap in the Energy Spectrum and the Thermodynamical Behavior at Lower Temperatures}

When the external field is much larger than the lower critical field $H_{c 1}$, a considerable number of flux lines is threading the superconductor and a uniform depression of the ordering parameter $\Delta$ becomes appreciable. In order to investigate this situation we assume that the effect of flux lines can be replaced by that of an equivalent magnetic field. Using a similar procedure to that employed in the study of the field dependence of the gap parameter in thin films [12], we obtain the following expression for $\Delta$ at the absolute zero of temperature

$$
\ln \Delta / \Delta_{\infty}=-\frac{\pi}{4} \zeta, \quad \text { for } \zeta \leq 1
$$

where

$$
\zeta=\frac{\tau_{t r} v^{2}}{3 \Delta} \overline{\left(2 e Q^{\prime}\right)^{2}}
$$

and

$$
Q^{\prime}=\frac{a}{2 \mathrm{e}} \sum_{m \neq 0} K_{1}\left(\alpha\left|r-r_{m}\right|\right) .
$$

We average $\left(Q^{\prime}\right)^{2}$ over a single cell with flux line at $r=0$, after subdividing the bulk into equivalent cells (hexagonal in the case of a triangular lattice) each containing a flux line. The current is given as [8]

$$
j=-\frac{2 \mathrm{e}^{2} N}{m} \tau_{t r} \Delta(\pi / 2-2 / 3 \zeta) A, \quad \text { for } \zeta \leq 1
$$

From this we find $a$ in Equation 33 to be

$$
a=\left\{\frac{4 \pi^{2} \tau_{t r} e^{2} N}{m} \Delta\left(1-\frac{4}{3 \pi} \zeta\right)\right\}^{1 / 2} .
$$

Similar calculations to those of Section 3 lead us to the following expression for the free energy

$$
\begin{aligned}
F & =F_{1}-\frac{1}{4 \pi} B \cdot H-\frac{m p_{0}}{4 \tau_{c}^{2}} \Delta^{2}\left(1-\frac{\pi}{2} \zeta+\frac{2}{3} \zeta^{2}\right), \\
F_{1} & =n \epsilon^{\prime}+\frac{n}{2}\left(\frac{a}{2 e}\right)^{2} \sum_{t^{2}+m^{2}+l m \geq 1} K_{0}\left(a \sqrt{\frac{2 \tau_{c}\left(1^{2}+m^{2}+1 m\right)}{\sqrt{3} e B}}\right) . \\
n & =\frac{e}{\pi} B, \quad \text { for a triangular lattice, }
\end{aligned}
$$

and

$$
\epsilon=\frac{m p_{0}}{12} \tau_{t r} v^{2} \Delta\left(1-\frac{4}{3 \pi} \zeta\right) \ln \left(\kappa_{3} \frac{\left(1-\frac{\pi}{4} \zeta\right)^{1 / 2}}{\left(1-\frac{4}{3 \pi} \zeta\right)}\right)
$$


In the last term we find the effect of uniform depression of $\Delta$ explicitly. The magnetic induction is obtained from

$$
\frac{\partial F}{\partial B}=0
$$

The above equation is apparently too difficult to be solved explicitly. When the external field is just above $H_{\mathrm{c} 1}$ and the flux lines are mutually fairly well separated, we find

$$
\zeta=\frac{\tau_{t r} v^{2}}{3 \Delta} \frac{36 \sqrt{3}}{2 \pi} e B e^{-2 a \sqrt{\frac{2 \pi}{\sqrt{3} e B}}}
$$

for a triangular lattice. We do not describe the derivation of the above equation, which is not very interesting. On the other hand when $H$ is much larger we have

$$
\zeta=\frac{\tau_{t r} v^{2}}{3 \Delta} \mathrm{e} B
$$

It is easy to show that the above expression is exact in the limit of vanishing $\Delta$, as is seen from the discussion given in $\mathrm{I}$. We expect that Equation (40) is valid in a much wider range of field, since Equation (39) gives a comparable value with Equation (40) when $B=0.1 \kappa^{-1} H_{c}$.

Using the expression (40), we will give a qualitative discussion on the thermodynamical behavior. In the presence of a field the gap in the energy spectrum $\omega_{0}$ is not always equivalent to the ordering parameter $\Delta$ but may be expressed as

$$
\omega_{0}=\Delta\left(1-\zeta^{2 / 3}\right)^{3 / 2}
$$

and consequently the specific heat at low temperatures is found to take [13]

$$
C_{s}(T)=\frac{m p_{0}}{\pi^{2}} \sqrt{\frac{2 \pi}{3}} T^{-1 / 2} \zeta^{-3 / 2} \Delta^{-1 / 3} \omega_{0}{ }^{\frac{11}{6}} e^{-\omega_{0 / T}}{ }^{*}
$$

where $\Delta$ and $\omega_{0}$ are calculated from

$$
\ln \Delta / \Delta_{\infty}=-\frac{\pi}{12} \frac{\tau_{t r} v^{2}}{\Delta} e B
$$

and

$$
\omega_{0}=\left\{\Delta^{2 / 3}-\left(\frac{\tau_{t r} v^{2}}{3} \mathrm{e} B\right)^{2 / 3}\right\}^{3 / 2} .
$$

If one takes account of the effect of the collective oscillation of the flux lines, a linear term in $T$ appears in the expression for the specific heat, which may dominate at extremely low temperatures.

In the vicinity of the second transition point where $\sqrt{\overline{|\Delta|^{2}}} / \pi T_{\text {co }}$ is small, the above expression is no longer valid. In this case the specific heat in a fixed external field is calculated by the use of the expression for the free energy (Equation (20) in I);

$$
F=\Delta F-\frac{1}{4 \pi} B \cdot H=-\frac{1}{4 \pi} \cdot \frac{H_{\mathrm{c} 2}(T)\left(H_{\mathrm{c} 2}(T)-H\right)}{\left(2 \kappa_{2}^{2}(T)-1\right)},
$$

*'The erratum found in the expression of Ref. 9 is corrected here. 
we obtain

$$
\begin{aligned}
C_{s}(T) & =T \frac{\partial^{2} F}{\partial T^{2}} \cong \frac{T}{4 \pi} \frac{2 H_{\mathrm{c} 2} \frac{\partial^{2} H_{\mathrm{c} 2}}{\partial T^{2}}}{\left(2 \kappa_{2}^{2}(T)-1\right) \beta} \\
& =\frac{m p_{0}}{3}\left\{\frac{2 \kappa_{1}^{2}(0)}{\left(2 \kappa_{2}^{2}(0)-1\right) \beta}\right\} T, \quad \text { for } T \ll T_{c o} \text { and } 2 \kappa_{2}^{2}>1 .
\end{aligned}
$$

From this we see that in the region $\sqrt{\mid \overline{|\Delta|^{2}}} / \pi T_{\text {co }} \ll 1$ where the magnetism $M$ is almost linear in the field, the linear term in $T$ predominates in the expression for the specific heat. As we have already noted in $I$, the expression (45) does not hold when $2 \kappa_{2}{ }^{2}>1$, where the phase transition at the second transition point becomes of the first order.

\section{Formulation: Inclusion of Pauli Spin Term}

In order to extend our previous formulation so as to include the effect of the Pauli paramagnetism, we first consider the impurity free case and begin with the following simple Hamiltonian [8]

$$
H=-\frac{1}{2 m} \int(i \nabla+\mathrm{e} A) \psi^{+} \cdot(i \nabla-\mathrm{e} A) \psi d x+g \int\left(\psi^{+} \psi\right)\left(\psi^{+} \psi\right) d x+\mu \int \psi^{+}(\sigma H) \psi d x,
$$

where the last term is the Pauli term. The generalized Gor'kov equations which include the Pauli term are the following:

$$
\begin{aligned}
& \left\{i \omega-\mu(\sigma H)-\frac{1}{2 m}(p-e A)^{2}+\zeta\right\} G_{\omega}\left(x, x^{\prime}\right)-i \Delta(x) F_{\omega}^{+}\left(x, x^{\prime}\right)=i \delta\left(x-x^{\prime}\right) \\
& \left\{i \omega-\mu(\sigma H)+\frac{1}{2 m}(p-e A)^{2}-\zeta\right\} F_{\omega}^{+}\left(x, x^{\prime}\right)+i \Delta^{+}(x) G_{\omega}\left(x, x^{\prime}\right)=0
\end{aligned}
$$

where $\zeta$ is the chemical potential and $G_{\omega}\left(x, x^{\prime}\right)$ and $F_{\omega}\left(x, x^{\prime}\right)$ are the fourier coefficients of Gor'kov's two Green's functions defined by

$$
\begin{aligned}
& G\left(x, x^{\prime}\right)=\left(\begin{array}{c}
-i<T \psi(x), \psi^{+}\left(x^{\prime}\right)> \\
-i<T \psi(x), \psi^{+}\left(x^{\prime}\right)>
\end{array}\right) \\
& F\left(x, x^{\prime}\right)=\left(\begin{array}{c}
<T \psi(x), \psi\left(x^{\prime}\right)> \\
<T \psi(x), \psi\left(x^{\prime}\right)>
\end{array}\right) .
\end{aligned}
$$

The ordering parameter $\Delta(x)$ is determined by the following equation

$$
\Delta(x)=\frac{1}{2}|g| \sum_{\omega}\left(F_{\omega}^{+}(x, x)+F_{\omega}^{-}(x, x)\right) .
$$

We are interested in the behavior of $\Delta(x)$ in the vicinity of the transition point where $\Delta$ is small $\left(\Delta / \pi T_{\text {co }} \ll 1\right)$. Expanding the above equations in powers of $\Delta(x)$ we obtain 


$$
\begin{aligned}
\Delta^{+}(x)=|g| T \sum_{n} & \frac{1}{2} S p \int G_{\omega}{ }^{\sigma}\left(r^{\prime}, r\right) G_{-\omega} \sigma\left(r, r^{\prime}\right) \Delta^{+}\left(r^{\prime}\right) d^{3} r^{\prime} \\
& -|g| T \sum_{n} \frac{1}{2} S p \iiint G_{\omega}^{\sigma}(s, r) G_{-\omega} \sigma(s, 1) G_{\omega}^{\sigma}(m, 1) G_{-\omega} \sigma(m, s) \cdot \Delta^{+}(s) \Delta(1) \Delta^{+}(m) d^{3} s d^{3} l d^{3} m
\end{aligned}
$$

$\omega_{n}=\pi T(2 n+1)$

where $G_{\omega}{ }^{\sigma}\left(r, r^{\prime}\right)$ is the spin dependent Green's function of an electron in a normal metal and the Fourier transform is given by

$$
\left\{i \omega-\mu(\sigma H)-\frac{1}{2 m}(p-e A)^{2}-\zeta\right\}^{-1}
$$

and $S p$ means the summation over spin states.

The effect of impurity scattering is included into the theory by renormalizing the self-energy and the vertex function as described in $I$. In the following we consider for simplicity the case where the spindependent part in the scattering amplitude from an impurity atom is inappreciable. In this case we arrive at the following equation

$$
\begin{aligned}
\left\{\ln T / T_{c o}+f_{0}\left[\frac{\tau_{t r} v^{2}}{12 \pi T}(i \nabla+2 e A)^{2}\right.\right. & \left.\left.+\frac{i \mu H}{2 \pi T}\right]\right\} \nabla^{+}(x) \\
& +\frac{1}{8(\pi T)^{2}} \operatorname{Re}\left\{\sum_{n}\left(n+\frac{1}{2}+\frac{i \mu H}{2 \pi T}\right)\left(\prod_{i=1}^{4} X_{n i}^{-1}\right)\right\} \Delta^{+}(1) \Delta(2) \Delta^{+}(3) \mid=0,
\end{aligned}
$$

$1=2=3=x$

where

$$
f_{0}(z)=\operatorname{Re}\left\{\psi\left(\frac{1}{2}+z\right)-\psi\left(\frac{1}{2}\right)\right\}
$$

and $\psi(z)$ is the di-gamma function.

The current is given as

$$
\begin{aligned}
j(x)= & -\left.\frac{e \tau_{t r} N}{4 \pi m T} \operatorname{Re}\left\{\sum_{n}\left(\prod_{i=1}^{2} X_{n i}^{-1}\right)\right\}\left(i \vec{\nabla}_{1}-i \vec{\nabla}_{2}+4 \mathrm{e} A\right) \Delta^{+}(1) \Delta(2)\right|_{1=2=x} \\
& -\frac{3 N \mu}{2 \pi m v^{2} T} \operatorname{rot}\left[\operatorname{lm}\left\{\sum_{n} \prod_{i=1}^{2} X_{n i}^{-1}\right\}|\Delta(x)|^{2}\right]
\end{aligned}
$$

where

$$
\left.X_{n i}=n+\frac{1}{2}+\frac{i \mu H}{2 \pi T}\left(\vec{q}_{i}-(-1)^{i} 2 e A\right)^{2}\right)
$$

and $q_{i}$ operates only on $\Delta(i)$.

Strictly speaking, on the right-hand side of Equation (53) we should add a constant term, (independent of $\Delta$ ) coming from the susceptibility due to normal Pauli paramagnetism. We neglect such a term in the 
present discussion, since the results are hardly affected by this term (possibly within $0.1 \%$ in the usual high field superconductors).

\section{The Upper Critical Field}

Before going into the discussion of Abrikosov's structure, let us consider the field corresponding to the lower instability limit of the normal state, which is obtained from the linear part of Equation (52)

$$
\left\{\ln \frac{T}{T_{\mathrm{co}}}+f_{0}\left[\frac{\tau_{t r} v^{2}}{12 \pi T}(i \nabla+2 e A)^{2}+\frac{i \mu H}{2 \pi T}\right]\right\} \Delta^{+}(\pi)=0 .
$$

In a uniform field $H$ along the $Z$ direction $(q-2 e A)^{2}$ is rewritten as

$$
-\frac{\partial^{2}}{\partial x^{2}}-\left(\frac{\partial}{\partial y}-2 i e H x\right)^{2}-\frac{\partial^{2}}{\partial z^{2}} \text {. }
$$

The eigenfunction and the eigenvalue are given by

$$
\Delta(x)=e^{-e H x^{2}},
$$

and

$$
\ln T / T_{\text {co }}+f_{0}\left(\frac{r_{t r} V^{2}}{6 \pi T} e H+\frac{i \mu H}{2 \pi T}\right)=0,
$$

respectively.

When the phase transition is of the second order the above field is just the upper critical field and we denote it by $H_{\mathrm{c} 2}^{*}$. Employing the asymptotic form of $\psi(z)$ we obtain.

$$
\begin{aligned}
H_{c 2}^{*} & =\frac{3 \Delta_{o o}}{2 e \tau_{t r} v^{2}}\left(1+a^{2}\right)^{-1 / 2}\left\{1-\frac{2}{3}\left(\frac{\pi T}{\Delta_{o o}}\right)^{2} \frac{1-a^{2}}{1+a^{2}}\right\}, \quad \text { for } T \ll T_{c o}, \\
& =\frac{12 T_{c o}}{\pi e \tau_{t r} v^{2}} \theta\left\{1-\theta\left(\frac{1}{2}-\frac{28 \zeta(3)}{\pi^{4}}\left(1-a^{2}\right)\right)\right\}, \quad \text { for }\left|T_{c o}-T\right| \ll T_{c o},
\end{aligned}
$$

where

$$
a=\frac{3 \mu}{e \tau_{t r} v^{2}}=\frac{\sqrt{2} H_{c 2}(0)}{H_{c p}(0)} \quad \text { and } \quad \theta=1-\frac{T}{T_{c o}}
$$

$H_{c 2}$ is the usual upper critical field in the absence of the Pauli term and calculated in $I$ and $H_{c p}=\frac{\Delta_{o o}}{\sqrt{2} \mu}$ as defined by Clogston, corresponding to the field where the energy difference due to the Pauli susceptibility becomes sufficient to destroy the superconducting correlation.

From the above expressions we see that the effect of the Pauli paramagnetism is appreciable at lower temperatures. Especially, at $T=0^{\circ} \mathrm{K}$ we have

$$
\begin{aligned}
H_{c 2}^{*} & =\frac{3 \Delta_{c o}}{2 e \tau_{t r} v^{2}}\left(1+a^{2}\right)^{-1 / 2}, \\
& =\frac{H_{c 2} H_{c p}}{\sqrt{2 H_{c 2}^{2}+H_{c p}^{2}}} .
\end{aligned}
$$


On the other hand, when the phase transition is of the first order, as is usually the case at lower temperatures with superconductors having a large Pauli term $(\alpha>1.47)$, the above field is a supercooling field. In

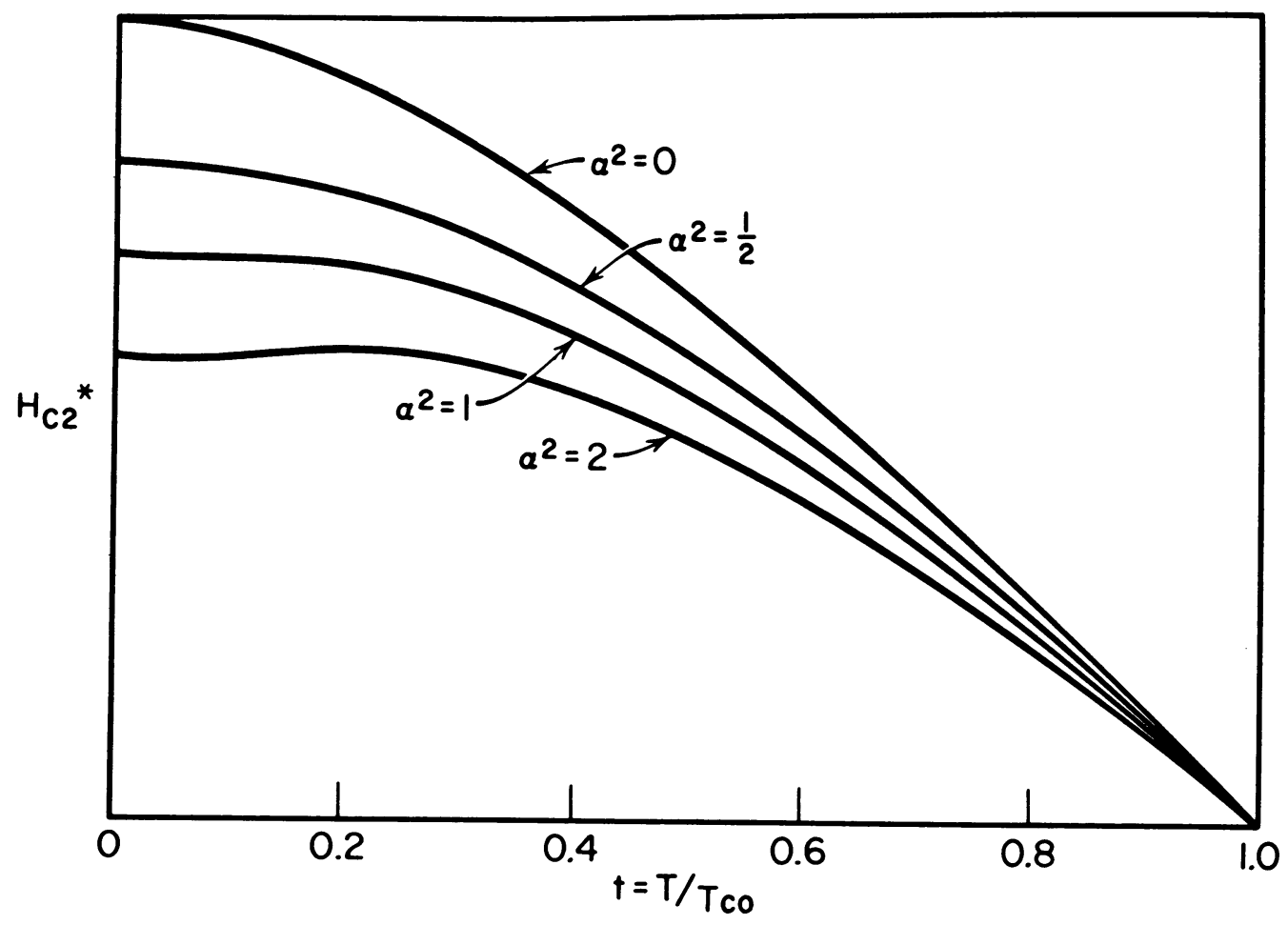

FIGURE 2

The upper critical field $H_{\mathrm{c} 2}^{*}$ as a function of temperature for $a^{2}=0,1 / 2,1$, and 2 .

this case it requires an explicit knowledge of the coefficient of $\Delta^{6}$ in Equation (52) to determine the upper critical field. (See $\S 8$.) The curves of the upper critical field with temperature are depicted in Fig. 2 for
various $a$.

\section{Abrikosov's Structure}

When the magnetic field is close to $H_{\mathrm{c} 2}^{*}$ and the ordering parameter small, we can discuss the modification of Abrikosov's structure through the Pauli term using Equations (52) and (53).

According to Abrikosov [4] we solve the equation by the use of a variational function of the form

$$
\Delta(x)=\sum_{n=-\infty}^{\infty} C_{n} \mathrm{e}^{i k n y} \exp \left\{-\mathrm{e} H_{0}\left(x-\frac{n k}{2 \mathrm{e} H_{0}}\right)^{2}\right\} .
$$

where $H_{0}$ is the external field.

Inserting the above expression for $\Delta$ in Equation (53) and combining with the Maxwell equation

$$
\operatorname{rot} \operatorname{rot} A=4 \pi j
$$

we have

$$
\frac{\partial A_{y}}{\partial x}=H=H_{0}-\frac{e \tau_{t r} N}{m T} g(\rho)|\Delta|^{2},
$$


where

$$
g(\rho)=\operatorname{Re}\left\{\sum(1+i a) \frac{1}{\left(n+\frac{1}{2}+\rho\right)^{2}}\right\} \text {, }
$$

and

$$
\rho=\frac{\tau_{t r} v^{2}}{6 \pi T} e H_{0}(1+i a)
$$

Substituting Equations (61) and (63) in Equation (52) we obtain

$$
\frac{\tau_{t r} e v^{2}}{6 \pi T}\left(H_{c 2}-H_{0}\right) g(\rho) \overline{|\Delta|^{2}}+\frac{1}{8(\pi T)^{2}}\left\{\frac{4 \pi N}{3 m}\left(\mathrm{e} \tau_{t r} v g(\rho)\right)^{2}-f_{1}(\rho)\right\} \overline{|\Delta|^{4}}=0,
$$

where

$$
f_{1}(\rho)=\operatorname{Re}\left\{\sum \frac{1}{\left(n+\frac{1}{2}+\rho\right)^{3}}-\frac{\rho}{1+i a} \sum \frac{1}{\left(n+\frac{1}{2}+\rho\right)^{4}}\right\} .
$$

Putting $\beta=\overline{|\Delta|^{4}} /\left(\overline{|\Delta|^{2}}\right)^{2}$ we calculate the magnetic induction as well as the free energy as

$$
B=\bar{H}=H_{0}-\frac{H_{c 2}^{*}-H_{0}}{\left(2 \kappa_{2}^{* 2}-1\right) \beta},
$$

and

$$
\Delta F=\frac{1}{4 \pi}\left[B^{2}-\frac{\left(H_{c 2}^{*}-B\right)^{2}}{\left\{\left(2 \kappa_{2}^{* 2}-1\right) \beta+1\right\}}\right]
$$

where

$$
\kappa_{2}^{*}=\left(\frac{3 m f_{1}(p)}{8 \pi N}\right)^{1 / 2}\left(e \tau_{t r} v \cdot g(p)\right)^{-1}
$$

The asymptotic forms are:

$$
\begin{aligned}
\kappa_{2}^{*} & =\kappa_{2}(0)\left(1+a^{2}\right)^{-3 / 2}\left(1+6 a^{2}-3 a^{4}+\frac{4}{3} \frac{5-65 a^{2}+97 a^{4}-3 a^{6}}{1+a^{2}}\left(\frac{\pi T}{\Delta_{o \circ}}\right)^{2}\right)^{1 / 2}, \text { for } T \ll T_{c o}, \\
& =\kappa\left[1+\left(\frac{28 \zeta(3)}{\pi^{2}}\left(1-a^{2}\right)-\frac{30 \zeta(4)}{7 \zeta(3)}\right) \frac{2}{\pi^{2}} \theta\right], \quad \text { for } T_{\text {co }}-T \ll . T_{\infty},
\end{aligned}
$$

where $\kappa_{2}(0)$ is found in I.

The magnetization $M$ is derived from Equation (67) as

$$
-4 \pi M=\left(H-H_{c 2}^{*}\right)^{2}\left(\beta\left(2 \kappa_{2}^{* 2}-1\right)\right)^{-1} .
$$

The change of $\kappa_{2}^{*}$ with temperature is plotted in Fig. 3. 


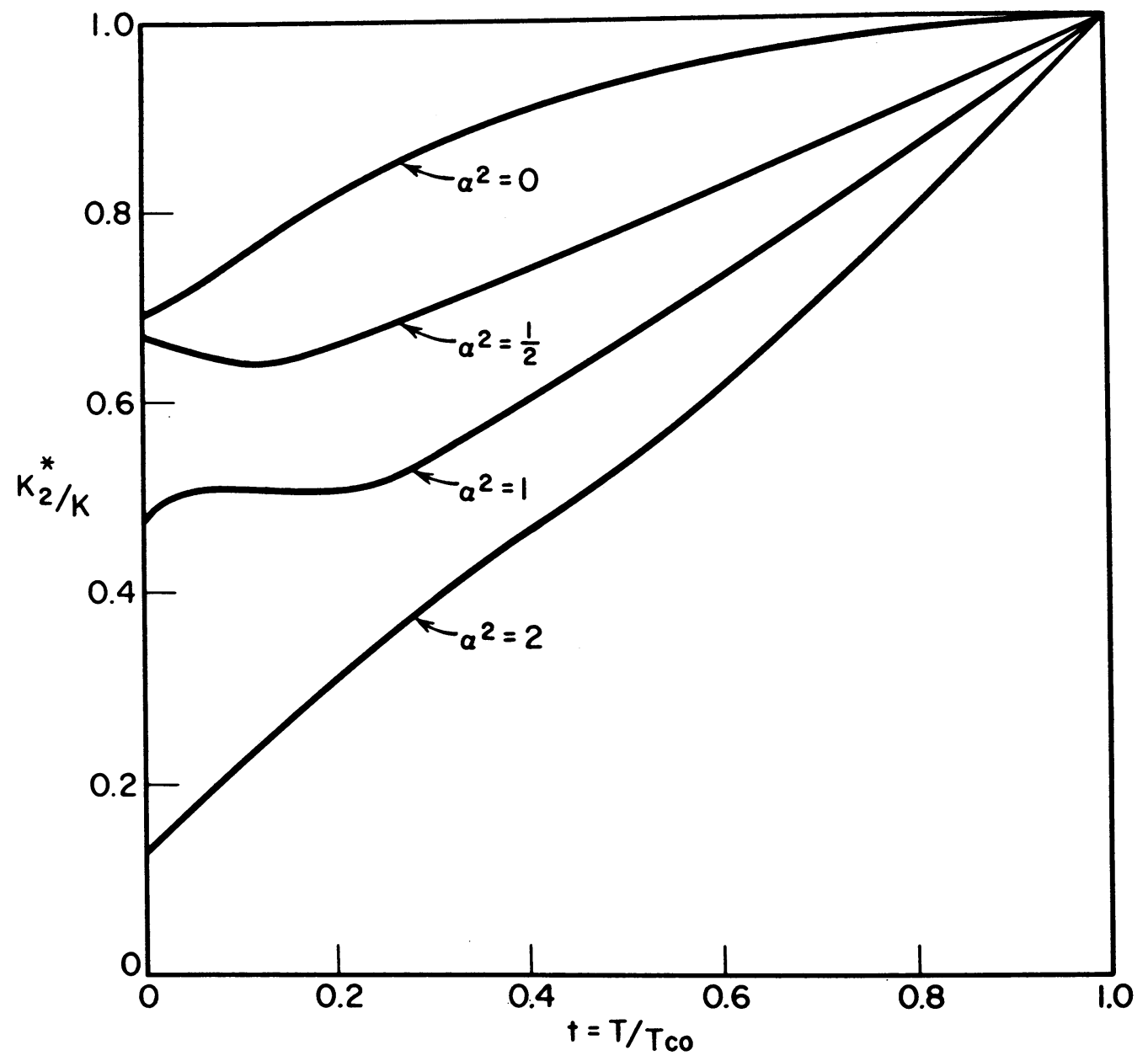

FIGURE 3

$\kappa_{2}^{*}$ as a function of $T / T_{\text {co }}$ for $\alpha^{2}=0,1 / 2,1$, and 2 .

The specific heat in a fixed external field is computed in terms of $\kappa_{1}^{*}$ and $\kappa_{2}^{*}$ as given in I, where $\kappa_{1}^{*}$ is defined as $\kappa_{1}^{*}=H_{c 2}^{*} / \sqrt{2} H_{c}$.

$$
C_{s}(T)=T \frac{\partial^{2}}{\partial T^{2}}\left\{-\frac{1}{4 \pi} \frac{H_{c 2}(T)\left(H_{c 2}(T)-H_{0}\right)}{\left(2 \kappa_{2}^{* 2}(T)-1\right) \beta}\right\},
$$

and asymptotically

$$
\begin{aligned}
& =\frac{m p_{0}}{3}\left\{\frac{2 \kappa_{1}^{*}(T) \kappa_{1}^{*}(0)}{\left(2 \kappa_{2}^{* 2}(T)-1\right) \beta}\right\}\left(\frac{1-a^{2}}{1+a^{2}}\right) T, \quad \text { for } T \ll T_{c o}, \\
& =\frac{m p_{0}}{3}\left\{\frac{2 \kappa^{2}}{\left(2 \kappa^{2}-1\right) \beta}\right\}\left(\frac{48}{7 \zeta(3)}\right) T, \quad \text { for } T_{\mathrm{co}}-T \ll T_{\mathrm{co}} .
\end{aligned}
$$

From the general discussion of the second order phase transition given in I we see immediately that the order of the phase transition changes from the second to the first when $\kappa_{2}^{*}$ becomes smaller than $1 / \sqrt{2}$. In this case the above expression for the specific heat is no longer valid. In superconductors having a large Pauli paramagnetism $\kappa$ is usually large $(\kappa \gg 1)$ and the above situation becomes possible only when $a^{2} \geq 1+\frac{2}{\sqrt{3}}$. 


\section{Thermodynamic Behavior in the Vicinity of the Critical Point}

It is interesting to see more closely the thermodynamical behavior of the mixed state in the vicinity of the critical point. For this purpose the explicit evaluation of the coefficient of $\overline{|\Delta|^{6}}$ in Equation (65) is necessary [14]. We restrict here our consideration to the case $\kappa \gg 1$ where the calculation is rather simple. In this case we can neglect the effect of the diamagnetic current which gives a contribution of the order of $\kappa^{-2}$ and find by a slight extension of the calculation given in $\S 2$.

$$
\left\{\ln T / T_{c o}+f_{0}(\rho)\right\} \overline{|\Delta|^{2}}+\frac{1}{8(\pi T)^{2}} f_{1}(\rho) \overline{|\Delta|^{4}}-\frac{3}{128(\pi T)^{4}} f_{2}(\rho) \overline{|\Delta|^{6}}=0
$$

where

$$
f_{2}(\rho)=\operatorname{Re}\left\{\sum_{n=0}^{\infty} \frac{1}{\left(n+\frac{1}{2}+\rho\right)^{5}}-\frac{3 \rho}{1+i a} \sum_{n=0}^{\infty} \frac{1}{\left(n+\frac{1}{2}+\rho\right)^{6}}\right\}
$$

The free energy is calculated as

$$
\begin{aligned}
F_{s}-F_{n} & =\int_{0}^{\sqrt{\overline{|\Delta|^{2}}}} \Delta^{2} d(1 /|g|) \\
& =-\frac{m p_{0}}{2 \pi^{2}} \int_{0}^{\sqrt{\overline{|\Delta|^{2}}}} \Delta^{2}\left(\frac{f_{1}(\rho)}{4} \frac{\beta \Delta}{(\pi T)^{2}}-\frac{3 f_{2}(\rho)}{32} \frac{\beta^{*} \Delta^{3}}{(\pi T)^{4}}\right) d \Delta \\
& =-\frac{m p_{0}}{2 \pi^{2}}\left(\frac{f_{1}(\rho)}{16} \beta \frac{\left(\overline{|\Delta|^{2}}\right)^{2}}{(\pi T)^{2}}-\frac{f_{2}(\rho)}{64} \beta^{*} \frac{\left(\overline{|\Delta|^{2}}\right)^{3}}{(\pi T)^{4}}\right),
\end{aligned}
$$

where

$$
\beta^{*}=\overline{|\Delta|^{6}} /\left(\overline{|\Delta|^{2}}\right)^{3} \text {. }
$$

In the present case $(\kappa \gg 1)$ the critical point $\left(T_{1}, H_{1}\right.$ in the $T-H_{c 2}^{*}$ diagram) is determined as the root of the following transcendental equations

$$
\left.\begin{array}{r}
\ln T / T_{\mathrm{co}}+f_{0}(\rho)=0 \\
f_{1}(\rho)=0
\end{array}\right\}
$$

Putting

$$
\ln T / T_{\mathrm{co}}+f_{0}(\rho)=\left(\frac{T-T_{c}}{T_{c}}\right) g^{*}(\rho)
$$

where $T_{\mathrm{c}}$ is the transition temperature in a given external field and $g^{*}(\rho)=\frac{\rho}{1+i \alpha} g(\rho)$, we solve Equation (76)

$$
\overline{|\Delta|^{2}}=-\frac{8(\pi T)^{2}}{3 \beta^{*} f_{2}(\rho)}\left(\sqrt{\beta^{2} f_{1}^{2}(\rho)+6 \beta^{*}\left(\frac{T-T_{c}}{T_{c}}\right) g^{*} f_{2}(\rho)}-\beta f_{1}(\rho)\right) .
$$

The free energy and the specific heat are found 


$$
\begin{aligned}
F_{s}-F_{n}=-\frac{m p_{0}}{2 \pi^{2}} \frac{(\pi T)^{2}}{54\left(-f_{2} \beta^{*}\right)^{2}} & \left(\sqrt{\beta^{2} f_{1}^{2}+6 \beta^{*}\left(\frac{T}{T_{c}}-1\right) g^{*} f_{2}}-\beta f_{1}\right)^{2} \\
& \times\left(2 \sqrt{\beta^{2} f_{1}^{2}+6 \beta^{*}\left(\frac{T}{T_{c}}-1\right) g^{*} f_{2}}-\beta f_{1}\right),
\end{aligned}
$$

and

$$
\Delta C_{s}(T) \cong m p_{0} \frac{2}{3}\left(g^{*}\right)^{2} T \frac{\sqrt{\beta^{2}{f_{1}}^{2}+6 \beta^{*}\left(\frac{T}{T_{c}}-1\right) g^{* f_{2}}}}{\left(\sqrt{\beta^{2} f_{1}{ }^{2}+6 \beta^{*}\left(\frac{T}{T_{c}}-1\right) g^{* f_{2}}}+\beta f_{1}\right)^{2}},
$$

respectively. When $T$ is far from $T_{1}$ we obtain

$$
\Delta C_{s}(T)=\frac{m p_{0}}{3} \frac{2\left(g^{*}\right)^{2}}{\beta f_{1}(\rho)} T,
$$

which coincides with the expression (28) in the limit $\kappa \longrightarrow \infty$. On the other hand at the critical point where $f_{1}(\rho)$ vanishes we have

$$
\Delta C_{s}(T) \cong m p_{0} \frac{2}{3}\left(g^{*}\right)^{2} T\left(6 \beta^{*}\left(\frac{T}{T_{c}}-1\right) g^{* f_{2}}\right)^{-1 / 2}
$$

which diverges at $T=T_{c}$.

When the transition becomes of the first order the upper critical field is determined by the condition $F_{s}-F_{n}=0$ or

$$
\overline{|\Delta|^{2}}=4(\pi T)^{2} \frac{\beta f_{1}(\rho)}{\beta^{*} f_{2}(\rho)}
$$

Substituting this in Equation (76) we obtain

$$
\frac{\tau_{t r} \mathrm{e} v^{2}}{6 \pi T}\left(H_{\mathrm{c} 2}^{\prime}-H_{\mathrm{c} 2}^{*}\right) g(\rho)=-\frac{\beta^{2}}{8 \beta^{*}} \frac{f_{1}^{2}(\rho)}{f_{2}(\rho)},
$$

where $H_{\mathrm{c} 2}^{\prime}$ is the upper critical field and $H_{\mathrm{c} 2}^{*}$ is defined in Equation (58). Especially at lower temperatures we have

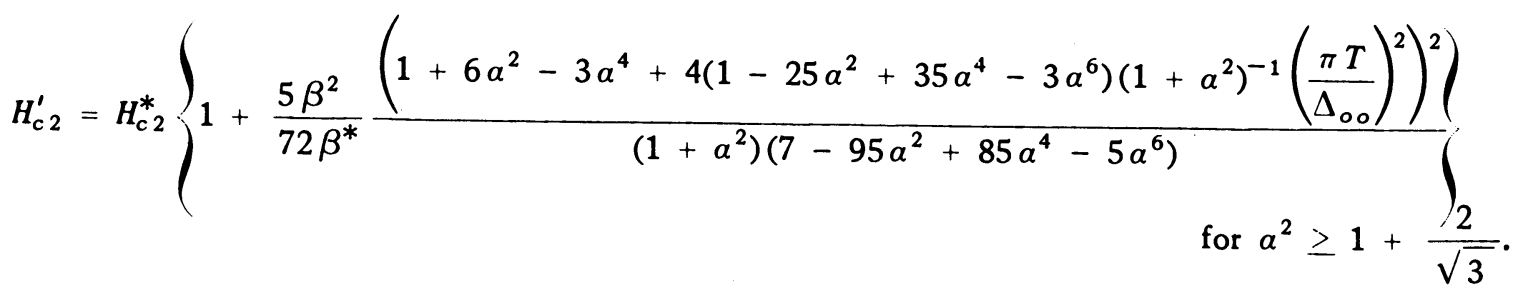

\section{Concluding Remarks}

We have seen thus far that Abrikosov's theory is valid in a wider region of both fields and temperatures if one uses appropriate parameters for $\kappa$ depending generally on the field and the temperature. In the present investigation we rely heavily on the fact that in superconducting alloys the magnetic field is introduced into the theory by simply replacing $\vec{q}$ by $\vec{q}+2 \mathrm{e} \vec{A}$ and it is not clear if Abrikosov's theory is valid in the above sense in the case of pure superconductors having large $\kappa$. 
As for the effect of Pauli paramagnetism we have seen that superconducting alloys having a large Pauli term are described by two parameters $\kappa_{1}^{*}$ and $\kappa_{2}^{*}$ without any difficulty as long as the phase transition is of the second order. We emphasize that the peculiar behavior pointed out in I for super conductors with $\kappa=\frac{1}{\sqrt{2}}$ is quite a common feature of superconductors having a large Pauli paramagnetism. In those superconductors the order of the transition from the mixed state into the normal state is the second in the vicinity of the transition temperature and becomes the first as temperature decreases. In this respect it is particularly important to have detailed experimental data on the magnetic as well as the thermodynamic properties at lower temperatures of high field superconductors.

\section{References}

1. K. MAKI, Physics 1, 21 (1964).

2. L. TEWORDT, Phys. Rev. 132, 595 (1963).

3. N. R. WERTHAMER, Phys. Rev. 132, 633 (1963).

4. A. A. ABRIKOSOV, JETP 32, 1442 (1957); Soviet Phys.-JETP 5, 1174 (1957).

5. B. S. CHANDRASEKHAR, Appl. Phys. Letters 1, 7 (1962).

6. A. M. ClOGSTON, Phys. Rev. Letters 9, 266 (1962).

7. R. R. HAKE and T. G. BERLINCOURT, Phys. Rev. 131, 140 (1963).

8. K. MAKI and T. TSUNETO, Prog. Theor. Phys. 31, 945 (1964).

9. K. MAKI and T. TSUNETO, Prog. Theor. Phys. 28, 163 (1962). Equation (2) is obtained from the consideration of the $S$ wave collective oscillation.

10. A. A. ABRIKOSOV and L. P. GOR'KOV, JETP 36, 319 (1959); Soviet Phys.-JETP 9, 220 (1959).

11. J. BARDEEN, L. N. COOPER and J. R. SCHRIEFFER, Phys. Rev. 108, 73 (1957).

12. K. MAKI, Prog. Theor. Phys. 29, 603 (1963); 31, 731 (1964).

13. A. A. ABRIKOSOV and L. P. GOR'KOV, JETP 39, 1781 (1960); Soviet-Phys. 12, 1243 (1961).

14. L. D. LANDAU and E. M. LIFSHITZ, Statistical Physics, (English translation, Pergamon Press, 1958). 